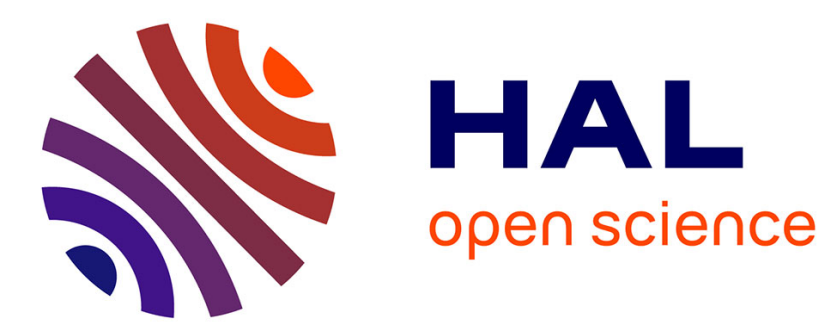

\title{
DoF in Sectored Cellular Systems with BS Cooperation Under a Complexity Constraint
}

\author{
Samet Gelincik, Michèle Wigger, Ligong Wang
}

\section{To cite this version:}

Samet Gelincik, Michèle Wigger, Ligong Wang. DoF in Sectored Cellular Systems with BS Cooperation Under a Complexity Constraint. 15th International Symposium on Wireless Communication Systems (ISWCS 2018), Aug 2018, Lisbonne, Portugal. 10.1109/ISWCS.2018.8491081 . hal-01905099

\section{HAL Id: hal-01905099 \\ https://hal.science/hal-01905099}

Submitted on 31 Aug 2021

HAL is a multi-disciplinary open access archive for the deposit and dissemination of scientific research documents, whether they are published or not. The documents may come from teaching and research institutions in France or abroad, or from public or private research centers.
L'archive ouverte pluridisciplinaire HAL, est destinée au dépôt et à la diffusion de documents scientifiques de niveau recherche, publiés ou non, émanant des établissements d'enseignement et de recherche français ou étrangers, des laboratoires publics ou privés. 


\title{
DoF in sectored Cellular Systems with BS Cooperation Under a Complexity Constraint
}

\author{
Samet Gelincik*, Michèle Wigger*, and Ligong Wang ${ }^{\dagger}$ \\ ${ }^{*}$ LTCI, Telecom ParisTech, Université Paris-Saclay, 75013 Paris, France \\ ${ }^{\dagger}$ ETIS-Université Paris Seine, Université de Cergy-Pontoise, ENSEA, CNRS, Cergy-Pontoise, France
}

\begin{abstract}
The paper presents upper and lower bounds on the per-user degrees of freedom (DoF) of a sectored hexagonal cellular model where neighboring basestations (BS) can cooperate during at most $\kappa$ interaction rounds over backhaul links of capacities $\mu=\mu_{\mathrm{DoF}} \cdot \frac{1}{2} \log P$, with $P$ denoting the transmit power at each mobile user. The lower bound is based on practically implementable beamforming and adapts the way BSs cooperate to the sector structure of the cells. It improves over the naive approach that ignores this sector structure. The upper bound is information-theoretic and holds for all possible coding schemes, including for example ergodic interference alignment whose practical implementation currently seems out of reach. Lower and upper bounds show that the complexity constraint imposed by limiting the number of interaction rounds $\kappa$ indeed limits the largest achievable DoF. In particular, irrespective of the backhaul capacity $\mu$, the per-user DoF cannot exceed a threshold which depends on $\kappa$.
\end{abstract}

\section{INTRODUCTION}

This paper considers the uplink of a hexagonal cellular network with multiple-antenna mobile users and multipleantenna basestations (BS). BSs are equipped with directional antennas, which allow them to create intracell sectors whose communications do not interfere. Following current standards, each cell is divided into three sectors, and each BS has the same number of antennas $M$ directed into any of the cell's sectors. The main quantity of interest in this paper is the degrees of freedom $(\mathrm{DoF})$ per user. The DoF of any generic [2] interference network with $M$-antenna transmitters and receivers is $M / 2$ [2]. Without cooperation, one needs interference alignment techniques, which are currently not implementable in practical systems, to achieve this DoF. When the BSs can cooperate over backhaul links, DoF $M / 2$ can be achieved also with more practical coding techniques such as one-shot interference alignment [2] combined with successive interference cancellation [5]. In fact, by means of backhaul cooperation, receiving BSs can pass their decoded messages to neighboring BSs according to a predefined decoding order of the BSs. The problem with this proposed successive interference cancellation scheme is its excessively long decoding delays stemming from the fact that BSs have to wait to decode their messages until all the preceding BSs have finished their decoding. To avoid such long delays, in this paper we restrict the number of interaction rounds between BSs.

Cooperation in communication networks was first considered in [9], and subsequently in many other works such as [5][8]. Previous results such as [9] show that for small networks, it suffices that cooperation takes place over a small number of interaction rounds. This is not the case for large networks, where performance generally improves with increasing number of interaction rounds [8].

The main interest of this paper is to understand the largest DoF that is achievable on the uplink when BS cooperation is limited in capacity and in the number of interaction rounds. For a lower bound, we propose a coding scheme that deactivates some of the mobile users in a way that the network decomposes into subnets, and then uses the backhaul links between BSs to collect quantized versions of the received signals within such a subnet at a single BS. This BS jointly decodes all the subnet's receive signals and distributes the decoded messages over the backhaul links to their intended BSs. The number of maximum allowed BS-interaction rounds $\kappa$ limits the size of the subnets, because each receive antenna in the subnet needs to be reachable in $\kappa / 2$ hops from one given BS to ensure that this BS can learn all the receive signals in the subnet and inform all the subnet's BSs about their decoded messages. The decoding in our scheme is thus reminiscent of clustered decoding as performed in [3], [4].

The new lower bound shows that $\mathrm{DoF} M / 2$ is achievable in the sectored hexagonal cellular model without interference alignment and with only two BS-interaction rounds and a backhaul DoF of $M / 6$.

The upper bound presented in this paper is informationtheoretic and allows for any coding scheme. The bound shows that for small backhaul capacities, the DoF of the proposed scheme is close to optimal, and that, irrespective of the capacities of the backhaul links, for finite $\kappa$, the DoF is strictly smaller than $M$.

\section{Problem DEFINITION}

\section{A. Network Model}

Consider the uplink communication of a cellular network consisting of $N \gg 1$ hexagonal cells as depicted in Figure 1. Each cell contains a BS equipped with $3 M$ directional receive antennas, with $M$ antennas covering each of the three sectors of the cell. Usage of directional antennas, where side lobe radiation patterns are negligible, implies that communications in the three sectors of a cell do not interfere with each other. This leads to the interference graph in Figure 1, where each sector is depicted by a small circle, and interfering sectors in different cells are connected by solid black lines. (Noninterfering sectors in the same cell are unconnected.) 


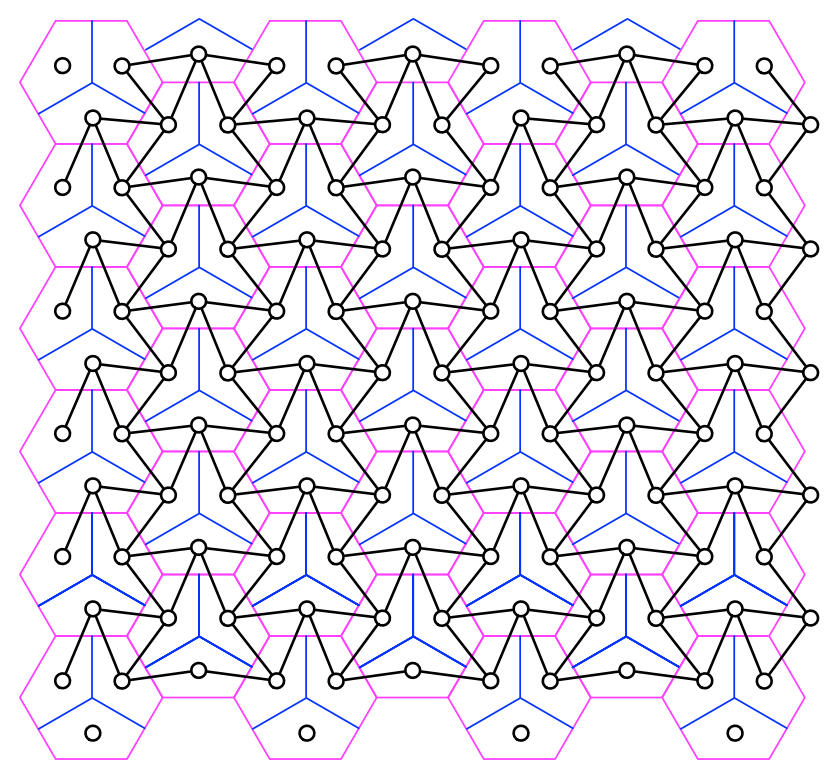

Fig. 1: sectored cellular model. Magenta hexagonal regions depict the various cells, and the blue lines depict sectors. Each circle depicts a mobile user that is equipped with $M$ transmit antennas. Each cell is associated to a BS such that each of the three sectors has a set of $M$ receive antennas directed to that sector.

Mobile users in a sector perform orthogonal multiple-access as is typical for current $4 \mathrm{G}$ networks, and we thus restrict our model to a single mobile user per sector. For simplicity and symmetry, we assume that each mobile user is equipped with $M$ transmit antennas. The uplink communication is then modeled by the following discrete-time input-output relation:

$\mathbf{Y}_{u, t}=\mathbf{H}_{u, u} \mathbf{x}_{u, t}+\sum_{\substack{v: \text { sector } v \\ \text { interferes sector } u}} \mathbf{H}_{u, v} \mathbf{x}_{v, t}+\mathbf{Z}_{u, t}, \quad t \in\{1, \ldots, n\}$,

where

- $u \in\{1, \ldots, 3 N\}$ denotes the index of a given cell-sector;

- $\mathbf{x}_{u, t}$ denotes the real-valued ${ }^{1} M$-dimensional time- $t$ signal sent by mobile user $u$;

- $\mathbf{Y}_{u, t}$ denotes the real-valued $M$-dimensional time- $t$ signal received at the $M$ BS receive antennas directed to sector $u$;

- $\mathbf{Z}_{u, t}$ denotes the real-valued $M$-dimensional i.i.d. standard Gaussian noise vector corrupting the time- $t$ signal at user $u$; it is independent of all other noise vectors;

- and $\mathbf{H}_{u^{\prime}, u}$ denotes a real-valued $M$-by- $M$ random matrix with entries that are independently drawn according to a standard Gaussian distribution, which models the channel from mobile user $u$ to the receive-antennas directed to sector $u^{\prime}$.

\footnotetext{
${ }^{1}$ Setup and results are easily generalized to complex-valued inputs and outputs.
}

Channel matrices are randomly drawn but assumed constant over the $n$ channel uses employed for the transmission of a message. In other words, the blocklength of a transmission is assumed shorter than the coherence time of the channel.

\section{B. Communication Model with Backhaul Cooperation}

Consider the uplink communication over the cellular network described in the previous section where each mobile user $u \in\{1, \ldots, 3 N\}$ wishes to send an independent message $W_{u}$, that is uniformly distributed over $\left\{1, \ldots,\left\lfloor 2^{n R_{u}}\right\rfloor\right\}$, to the BS in its cell. Communication takes place in two phases. In the first phase, each mobile user $u$ applies an encoding function $f_{u}^{(n)}:\left\{1, \ldots,\left\lfloor 2^{n R_{u}}\right\rfloor\right\} \rightarrow \mathbb{R}^{M \times n}$ to its message $W_{u}$ and sends the $n$ resulting vectors

$$
\mathbf{X}_{u}:=\left(\mathbf{X}_{u, 1}, \ldots, \mathbf{X}_{u, n}\right)=f_{u}^{(n)}\left(W_{u}\right)
$$

as its inputs over the network. Each encoding function $f_{u}^{(n)}$ has to be chosen so that the input vectors satisfy an average input power constraint $P$ :

$$
\frac{1}{n} \sum_{t=1}^{n}\left\|\mathbf{X}_{u, t}\right\|^{2} \leq P \quad \text { with probability } 1 .
$$

The second communication phase takes place after each receiving BS $j \in\{1, \ldots, N\}$ has observed all outputs at the antennas in each of the three sectors of the cell. It is over the backhaul links connecting adjacent BSs. The backhaul links are assumed to be noise-free but rate-limited, and can be interactive. However, to limit complexity and latency of the backhaul communications, we restrict the interaction to only $\kappa$ rounds, for a given positive integer $\kappa$.

For each $j \in\{1, \ldots, N\}$, let $\mathbf{Y}_{j}$ denote all the wireless signals observed at the $3 M$ antennas of $\mathrm{BS} j$ :

$$
\begin{aligned}
\mathbf{Y}_{j}:= & \left(\mathbf{Y}_{u_{j, 1}, 1}, \ldots, \mathbf{Y}_{u_{j, 1}, n}, \mathbf{Y}_{u_{j, 2}, 1}, \ldots, \mathbf{Y}_{u_{j, 2}, n}\right. \\
& \left.\mathbf{Y}_{u_{j, 3}, 1}, \ldots, \mathbf{Y}_{u_{j, 3}, n}\right)
\end{aligned}
$$

where $u_{j, 1}, u_{j, 2}$ and $u_{j, 3}$ denote the three sectors in sector $j$. In the $k$ th cooperation round, $k \in\{1, \ldots, \kappa\}$, each BS $j \in$ $\{1, \ldots, N\}$ calculates the message $V_{j \rightarrow i}^{k}$ that it sends to a neighboring BS $i$ as:

$$
V_{j \rightarrow i}^{k}=\psi_{j \rightarrow i}^{k,(n)}\left(\mathbf{Y}_{j}, \mathbf{V}_{\text {to } j}^{k-1}\right),
$$

for some cooperation functions $\left\{\psi_{j \rightarrow i}^{k,(n)}\right\}$ on appropriate domains. Here, $\mathbf{V}_{\text {to } j}^{k-1}$ denotes the $6 \cdot(k-1)$ cooperation messages BS $j$ has received from its 6 neighboring BSs during the first $k-1$ cooperation rounds:

$$
\begin{array}{r}
\mathbf{V}_{\text {to } j}^{k-1}:=\left\{V_{i^{\prime} \rightarrow j}^{k^{\prime}}: \text { BS } i^{\prime} \text { adjacent to BS } j\right. \\
\text { and } \left.k^{\prime}=1, \ldots, k-1\right\} .
\end{array}
$$

The cooperation functions have to be chosen in such a way that, for each pair of neighboring BSs $(i, j) \in\{1, \ldots, N\}^{2}$, the total information sent over the backhaul link from BS $j$ to BS $i$ is of rate not exceeding a given $\mu>0$ :

$$
\frac{1}{n} \sum_{k=1}^{\kappa} H\left(V_{j \rightarrow i}^{k}\right) \leq \mu \text {. }
$$


Once the BS-cooperation phase is terminated, each BS $j \in$ $\{1, \ldots, N\}$ proceeds to decode the three messages sent by the mobiles in the three sectors of its cell. To this end, for each sector $u$ in its cell, it applies a decoding function $h_{u}$ on corresponding domains to produce the message estimates

$$
\hat{W}_{u}:=h_{u}\left(\mathbf{Y}_{j}, \mathbf{V}_{\text {to } j}^{\kappa}\right) \text {. }
$$

An error occurs in the communication unless, for every sector $u \in\{1, \ldots, 3 N\}$ :

$$
\hat{W}_{u}=W_{u} .
$$

\section{Capacity and Degrees of Freedom}

A rate-tuple $\left(R_{1}, \ldots, R_{3 N}\right)$ is said achievable, if for every $\epsilon>0$ and sufficiently large blocklengths $n$, there exist encoding, cooperation, and decoding functions $\left\{f_{u}^{(n)}\right\},\left\{\psi_{j, i}^{k,(n)}\right\}$, and $\left\{h_{j}^{(n)}\right\}$, such that

$$
\operatorname{Pr}\left[\bigcup_{u=1}^{3 N}\left\{\hat{W}_{u} \neq W_{u}\right\}\right] \leq \epsilon .
$$

The capacity region $\mathrm{C}(P, \mu, \kappa)$ is defined as the closure of the set of all achievable rate-tuples, and the sum capacity $\mathrm{C}_{\Sigma}(P, \mu, \kappa)$ as

$$
\mathrm{C}_{\Sigma}(P, \mu, \kappa):=\sup _{\substack{\left(R_{1}, \ldots, R_{3 N}\right) \\ \in \mathrm{C}(P, \mu, \kappa)}} \sum_{u \in\{1, \ldots, 3 N\}} R_{u} .
$$

In the high signal-to-noise ratio (SNR) regime, the quantity of interest is the degrees of freedom (DoF), which is defined as:

$$
\operatorname{DoF}\left(\mu_{\text {DoF }}, \kappa\right):=\lim _{N \rightarrow \infty} \lim _{P \rightarrow \infty} \frac{C_{\Sigma}\left(P, \mu_{\text {DoF }} \cdot \frac{1}{2} \log P, \kappa\right)}{3 N \cdot \frac{1}{2} \log P} .
$$

Notice that here the backhaul cooperation rate $\mu$ scales with the power constraint $P$, and thus with the sum-capacity $C_{\Sigma}$.

\section{Uplink SCHEME with COOPERATIVE BSS}

The scheme has a parameter $t$, which is a positive integer. Choose a set of master BSs to form a regular grid of equilateral triangles where the three master cells forming a specific triangle lie $2 t$ cell-hops away from each other. As we shall see, the proposed scheme takes place over $2 t$ cooperation rounds, and thus one is allowed to choose any $t \in\left\{1, \ldots,\left\lfloor\frac{\kappa}{2}\right\rfloor\right\}$.

For simplicity, we first present the scheme in the special case where $t=2$. The general case is treated later.

\section{A. Special case where $t=2$}

Consider the network in Figure 2, where the magenta cells indicate the master cells. We refer to cells that are adjacent to master cells as layer-1 cells, and the cells that are adjacent to layer- 1 cells and that are not master cells as layer- 2 cells. According to the way we choose the master cells, every cell is either a master cell, a layer- 1 cell or a layer- 2 cell.

Encoding: The mobile users in the network that are not shown in Figure 2 remain silent and do not transmit anything. Each of the remaining mobile users $u$ transmits its message

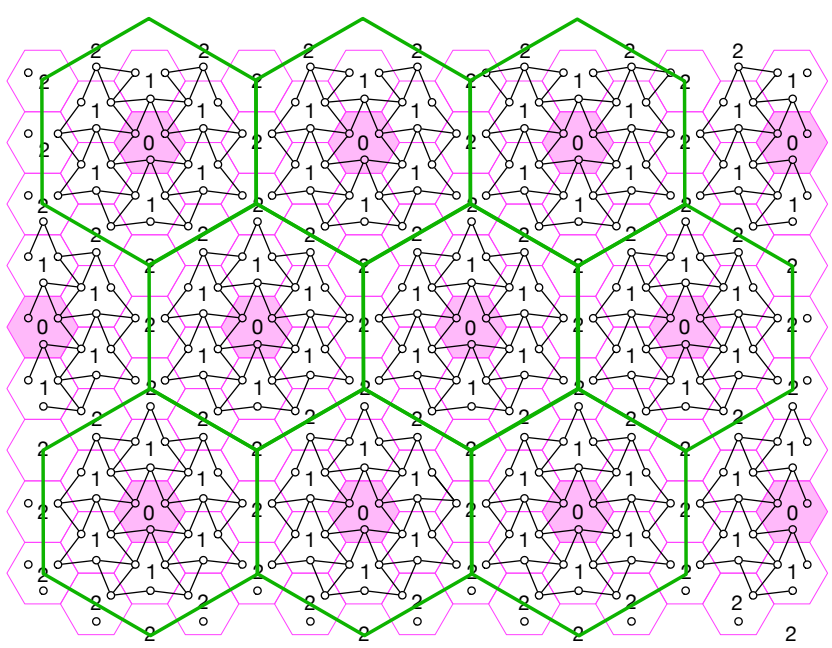

Fig. 2: Illustration of the network partition applied in our coding scheme for parameter $t=2$. Magenta cells denote master cells and green hexagonal regions illustrate the decoding regions. All messages sent within a green hexagonal region are decoded by the BS in the magenta cell at the center of that region.

$M_{u}$ using an independent Gaussian codebook of power slightly less than $P$.

Cooperation between BSs: Cooperation takes place over $2 t=4$ rounds as follows:

- Round 1: Each layer-2 BS applies an independent Gaussian vector quantizer to the $M$-dimensional receive signal of each of its sectors that contains an active mobile user. The quantizers are chosen to be of rate

$$
R_{q}=\frac{M}{2} \log (P) .
$$

Each layer-2 BS then sends the quantization message obtained for a given sector to one of the adjacent layer-1 BSs that lie in the same green hexagonal region as this sector, see Figure 2.

Each layer-1 BS applies a rate- $R_{q}$ Gaussian vector quantizer to each of its own three $M$-dimensional receive signals, and sends the three quantization messages to its adjacent master BS.

- Round 2: The layer-1 BSs forward the Round-1 messages they received from layer-2 BSs to their adjacent master BSs.

- Rounds 3 and 4: Each master BS uses the quantization messages received in Rounds 1 and 2 to reconstruct quantized versions of the $27 M$-dimensional received signals observed in the sectors lying in the same green hexagonal region as the master BS itself. Using these 27 quantized $M$-dimensional signals and its own $3 M$ dimensional receive signals, each master BS decodes all the messages sent by the 30 active mobile users in this green hexagonal region. 
In Rounds 3 and 4, messages decoded at the master BS but intended for layer-1 or layer-2 BSs are sent over the backhaul links to the intended BSs. Specifically, messages for layer-1 BSs are directly passed from the master BSs to their intended BSs in Round 3. Messages for layer-2 BSs are first passed to adjacent layer-1 BSs in Round 3, and then to the intended layer-2 BSs in Round 4.

Decoding: Each master BS declares its own message that it has produced during the decoding steps preceding Round 3. Each layer-1 BS declares the messages it has received in Round 3 and that were intended for it. Each layer-2 BS declares the messages that it has received in Round 4.

Analysis: Our interest is in the high-SNR asymptotics, i.e., in the DoF. Choosing the quantization rate $R_{q}$ as in (10) implies that each received signal is essentially quantized at the noise level, and thus allows us to achieve the same DoF as if the master BS had direct access to all the 30 receive signals in its decoding region. Since the channel matrix corresponding to the active sectors in a single green hexagonal region is of full rank with probability one, each of these 30 transmitted messages can be decoded with small probability of error at a DoF of $M$ per active user. Notice now that for large networks $(N \rightarrow \infty) \frac{5}{6}$ of the mobile users are active, and thus the scheme achieves

$$
\text { DoF }_{\text {scheme }}=\frac{5 M}{6} \text {. }
$$

We analyze the backhaul load for a single green hexagonal region. In Round 1, layer-2 BSs send in total 9 quantization messages, each of $M$ DoF, to layer-1 BSs, which in turn relay these messages to their adjacent master BS in Round 2. Additionally, in Round 1, layer-1 BSs send in total 18 quantization messages, each of $M$ DoF, to their adjacent master BSs. In Round 3, the master BS sends 27 decoded messages, each of DoF $M$, to the 6 adjacent layer- 1 BSs. Of these 27 decoded messages, 9 are forwarded to adjacent layer2 BSs in Round 4. Hence in total 54 cooperation messages of DoF $M$ are sent in each green hexagonal region.

The backhaul load in our scheme is unevenly distributed among backhaul links. To balance this load and to achieve fairness among the rates achieved by the various mobile users, instances of the presented scheme are time-shared, such that in different instances different cells play the role of master cells. We are then interested in the average backhaul DoF used by our scheme. To calculate this average backhaul DoF, notice that each green hexagonal region is associated with a single master cell, and a cluster of 11 neighboring cells is associated with each of the master cells. The average backhaul load per cooperation link is then

$$
\mu_{\text {DoF }}=\frac{54 M}{6 \cdot(11+1)}=\frac{3 M}{4},
$$

where the factor 6 accounts for the fact that each cell has 6 outgoing cooperation links.

\section{B. General case}

Choose the master cells so that they form a regular pattern of equilateral triangles where the master cells building these

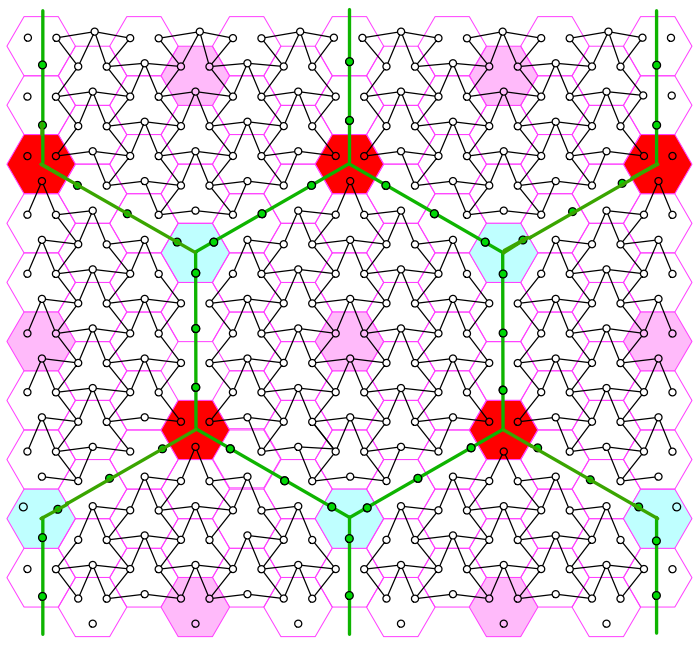

Fig. 3: Illustration of hexagon subnets for $t=3$. Pink cells, red cells and blue cells represent master cells, corner cells and null cells, respectively. Green-filled circles refer to silenced users.

triangles are $2 t$ cell-hops away from each other. (See Figure 3 for a choice of magenta master cells when $t=3$.) For $\ell=$ $1, \ldots, t$, we call layer- $\ell$ cells the cells that are at distance $\ell$ from the closest master cell. The distance between two cells here is defined as the minimum number of cell-hops needed to get from one cell to the other.

Encoding: Some of the layer- $t$ mobile users are deactivated and do not transmit any message at all. All other mobile users are active, and send their messages using an independent Gaussian codebook of power $P$.

To describe the mobile users that are deactivated, we introduce the following denomination. We call the cells that are located $t$ hops above the master cells the corner cells and the cells $t$ hops below the master cells the null cells. We silence all users of the null cells and none of the corner cells. For all layer- $t$ cells that are neither corner nor null cells, we deactivate the single mobile users that is closest to a corner cell. We conclude that among all the layer- $t$ cells that surround a master cell, 3 of them have only active users, 3 have only deactivated users, and the remaining $6(t-1)$ cells have 2 active users. See Figure 3 for an example.

Cooperation between BSs: Cooperation takes place over $2 t$ rounds.

- Rounds 1 to $t$ : Each BS that is not a master BS applies a rate- $R_{q}$ Gaussian vector quantizer to each of its $M$ dimensional receive signals that corresponds to a sector with an active mobile user. For $\ell=1, \ldots, t-1$, each layer- $\ell$ BS sends its 3 quantization messages to the closest master BS by means of multi-hop communication over $\ell$ rounds. Each layer- $t$ BS sends each of its produced quantization messages to the master BS that is closest to the sector of each of the quantized signals. This transmission is again multi-hop over $t$ rounds.

- Rounds $t+1$ to $2 t$ : Each master BS reconstructs the $M$-dimensional quantized signals corresponding to the 
quantization messages received in Rounds 1 to $t$. It then decodes the messages sent by the mobile users in the sectors corresponding to these quantization messages as well as the messages sent by the mobile users in its own cell. Finally, during cooperation rounds $t+1, \ldots, 2 t$, it communicates each of the decoded messages using multihop communication to its intended BS.

Decoding: Each master BS directly declares the messages guessed for its own cell before Round $t+1$. The other BSs declare the messages that the master BSs have guessed for their cells and sent to them during Rounds $t+1, \ldots, 2 t$.

Analysis: As before, the quantization rate $R_{q}$ is chosen at noise level, see (10). This allows us to achieve the same DoF as if the master BSs had direct access to all the receive signals for which they receive quantization information. Since there are $6 \ell$ layer- $\ell$ cells around each master cell, the number of quantization messages received by each master $\mathrm{BS}$ is

$$
3 \cdot \sum_{\ell=1}^{t-1} 6 \ell+(6 t-3)=9 t(t-1)+6 t-3=3\left(3 t^{2}-t-1\right) .
$$

Thus, including the 3 messages of the mobile users in its own cell, each master BS decodes $3 t(3 t-1)$ messages, each with a DoF equal to $M$.

For $N \gg 1$, a cell partitioning can be obtained such that each master BS is associated with a cluster that includes all the layer-1, layer- $2, \ldots$, layer- $(t-1)$ cells that are closest to this master cell; among the layer- $t$ cells, six are respectively shared between three clusters, while the rest are shared between two clusters, thus a total of $(3 t-1)$ layer- $t$ cells should be counted for each cluster. Including the master cell itself, the number of cells counted in such a cluster is thus

$$
1+\sum_{\ell=1}^{t-1} 6 \ell+(3 t-1)=3 t^{2}
$$

The scheme thus achieves a DoF of

$$
\mathrm{DoF}_{\text {scheme }}=\frac{M \cdot 3 t(3 t-1)}{3 \cdot 3 t^{2}}=\frac{(3 t-1) M}{3 t} .
$$

We next analyze the backhaul load that is associated with a single master cell. During Rounds 1 to $t$, a master BS receives a quantization message of DoF $M$ from each sector that has an active user and that is closer to this master BS than to any other master BS. Since the quantization message for a sector in layer- $\ell$ is communicated over $\ell$ backhaul links, the total backhaul DoF used in these first $t$ rounds is

$$
\begin{aligned}
& 3 M \sum_{\ell=1}^{t-1} 6 \ell \cdot \ell+M(6 t-3) t \\
& =3 M[(t-1) t(2 t-1)+(2 t-1) t] \\
& =3 M t^{2}(2 t-1) .
\end{aligned}
$$

During Rounds $t+1$ to $2 t$, a decoded message of DoF $M$ is communicated from the master BS to the corresponding BS for each quantization message that has been sent during Rounds 1 to $t$. Therefore, the total backhaul load during Rounds $t+1$

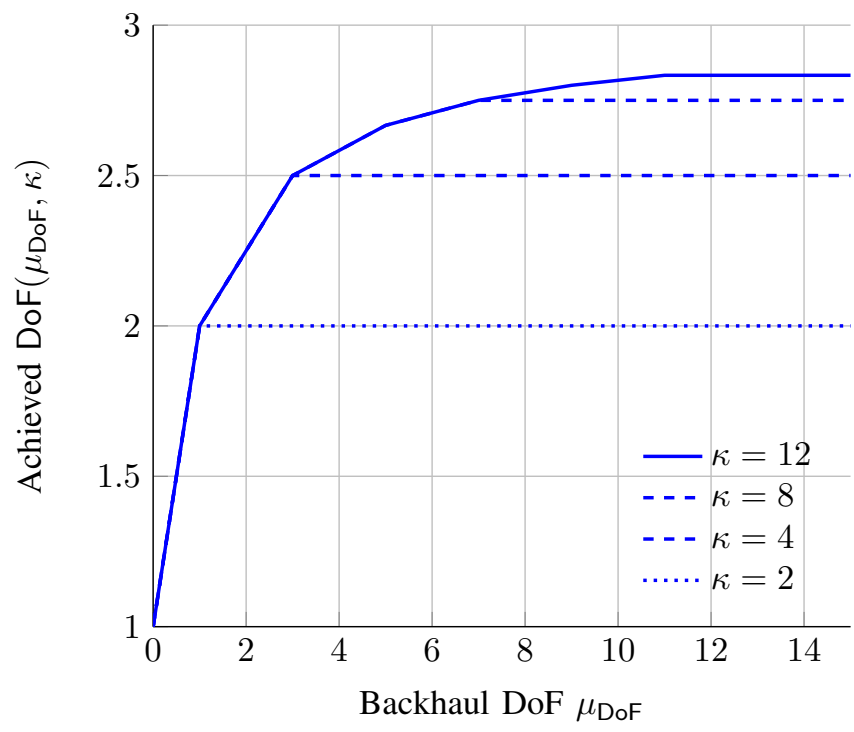

Fig. 4: Lower bound on the DoF in Theorem 1 as a function of $\mu_{\text {DoF }}$ when $M=3$ and for different values of $\kappa$.

to $2 t$ is equal to that during Rounds 1 to $t$. The total backhaul load over all rounds is thus

$$
6 M t^{2}(2 t-1) .
$$

To balance the backhaul load and to achieve fairness among the rates achieved by the various mobile users, the presented scheme is time-shared such that in different instances different cells play the role of the master cells. Under such a timesharing framework, the average backhaul DoF is the limiting quantity. The average backhaul load is obtained by dividing (17) by the number of outgoing (or incoming) backhaul links in a cluster, which by (14) is $6 \cdot 3 t^{2}$ :

$$
\mu_{\mathrm{DoF}}=\frac{6 M t^{2}(2 t-1)}{6 \cdot 3 t^{2}}=\frac{(2 t-1) M}{3} .
$$

\section{Tradeoff Between DoF and Backhaul Load}

Time-sharing the described scheme for different values of parameter $t$ yields the following result. Define $\mu_{\mathrm{DoF}, 0}=0$ and $\mathrm{DoF}_{0}=\frac{M}{3}$, and for each $t \in\left\{1, \ldots,\left\lfloor\frac{\kappa}{2}\right\rfloor\right\}$ :

$$
\mu_{\mathrm{DoF}, t}:=\frac{(2 t-1) M}{3} \text { and } \mathrm{DoF}_{t}:=\frac{(3 t-1) M}{3 t} .
$$

Theorem 1 (Achievability): Let $f_{\kappa}(\cdot)$ be the mapping from $\mu_{\text {DoF }}$ to DoF defined by the upper hull over the set

$$
\left\{\left(\mu_{\mathrm{DoF}, t}, \mathrm{DoF}_{t}\right): t=0, \ldots,\left\lfloor\frac{\kappa}{2}\right\rfloor\right\},
$$

then

$$
\operatorname{DoF}\left(\mu_{\mathrm{DoF}}, \kappa\right) \geq f_{\kappa}\left(\mu_{\mathrm{DoF}}\right) .
$$

Figure 4 illustrates this lower bound for different values of $\kappa$. Notice that even with only two conferencing rounds, a DoF of $M / 2$ is achievable with our scheme with a backhaul DoF of only $\mu_{\text {DoF }}=0.5$. 
A naive alternative to our scheme would be to ignore the interference pattern caused by the sector structure of the cells and to simply deactivate all mobile users in layer- $t$ cells. Compared to our scheme this naive approach reduces the DoF from $M\left(1-\frac{1}{3 t}\right)$ to $M\left(1-\frac{3 t+2}{3(t+1)^{2}}\right)$, and it reduces the number of required cooperation rounds from $2 t$ to $2(t-1)$ because the first and the last cooperation rounds of our protocol become useless when all layer- $t$ users are deactivated.

Define $\mu_{\text {naive }, 0}=0$ and $\mathrm{DoF}_{\text {naive }, 0}=\frac{M}{3}$, and for each $t \in$ $\left\{1, \ldots,\left\lfloor\frac{\kappa}{2}\right\rfloor\right\}$ :

$$
\begin{aligned}
\mu_{\text {naive }, t} & :=\frac{t(2 t+1) M}{3(t+1)} \\
\text { DoF }_{\text {naive }, t} & :=M\left(1-\frac{(3 t+2)}{3(t+1)^{2}}\right) .
\end{aligned}
$$

Let $g_{\kappa}(\cdot)$ be the mapping defined by the upper hull over the set

$$
\left\{\left(\mu_{\text {naive }, t}, \text { DoF }_{\text {naive }, t}\right): t=0, \ldots,\left\lfloor\frac{\kappa}{2}\right\rfloor\right\},
$$

then the DoF of this naive approach is given by

$$
\text { DoF }_{\text {naive }}=g_{\kappa}\left(\mu_{\text {naive }, t}\right) \text {. }
$$

We now present an upper bound on the DoF.

Theorem 2 (An information-theoretic converse): We have:

$$
\begin{aligned}
& \operatorname{DoF}\left(\mu_{\mathrm{DoF}}, \kappa\right) \\
& \leq \min \left\{\frac{M}{2}+\frac{2}{3} \mu_{\mathrm{DoF}}, M\left(1-\frac{1}{2\left(1+\kappa+\kappa^{2}\right)}\right)\right\} .
\end{aligned}
$$

Proof: See the extended version [1].

Remark that for any finite $\kappa$, DoF $\left(\mu_{\mathrm{DoF}}, \kappa\right)<M$, irrespective of the backhaul capacity $\mu_{\mathrm{DoF}}$.

Figure 5 compares the new lower bound in Theorem 1 with the lower bound achieved by the naive approach. The figure also shows the information-theoretic upper bound in Theorem 2. Notice that the lower bounds on the DoF are based on practically implementable coding schemes. In particular, interference alignment is not used, even though it is known to achieve a DoF of $M / 2$ over a generic network in the absence of BS cooperation. In contrast, the information-theoretic upper bound holds for any possible coding scheme. It is thus natural that there is a gap between the presented upper and lower bounds.

\section{CONCLUding Remarks}

The paper presents a lower bound on the DoF of the uplink of a sectored cellular network with backhaul cooperation between BSs. This DoF is characterized as a function of the backhaul capacity, and also of the maximum number of allowed cooperation rounds. The presented results show that limiting the number of cooperation rounds also limits the DoF achievable over the network. In particular, to get a DoF of $M$, an infinite number of cooperation rounds is required as the number of cells in the network grows. If the number of cooperation rounds does not grow with the number of cells but is bounded, then also the DoF is bounded away from $M$,

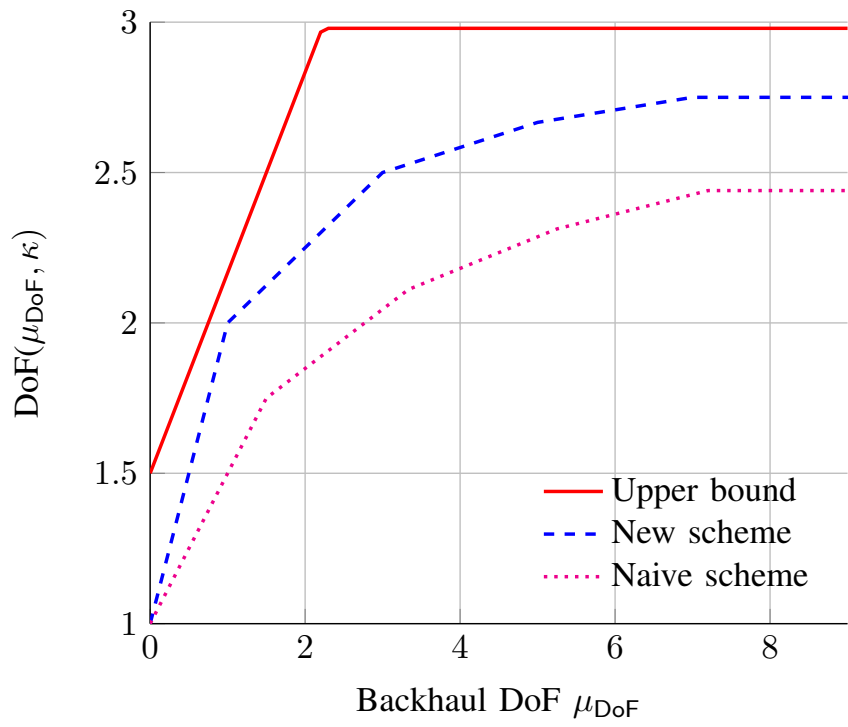

Fig. 5: Lower and upper bounds on the DoF as a function of $\mu_{\text {DoF }}$ for $M=3$ and $\kappa=8$.

even in the idealistic case of unlimited backhaul cooperation capacity.

The obtained lower bound on the DoF is based on a practically implementable scheme. Obtaining dual results for the downlink is straightforward. Finite-SNR results and information-theoretic converses are derived in [1].

\section{ACKNOWLEDGEMENT}

M. Wigger was supported by a Huawei Disruptive ICT Grant.

\section{REFERENCES}

[1] S. Gelincik, M. Wigger, and L. Wang, "Benefits of local cooperation in sectorized cellular models," in preparation, 2018.

[2] S. A. Jafar, "Interference alignment a new look at signal dimensions in a communication network," Found. and Trends in Com. and Inf. Theory, vol. 7, no. 1, pp. 1-134, 2011.

[3] G. Katz, B. M. Zaidel, and S. Shamai (Shitz), "On layered transmission in clustered cooperative cellular architectures," in Proc. 2013 IEEE ISIT, Istanbul, Turkey, Jul. 7-12, 2013, pp. 1162-1166.

[4] S.-J. Kim, S. Jain, and G. B. Giannakis, "Backhaul-constrained multi-cell cooperation using compressive sensing and spectral clustering," in Proc. 2012 SPAWC, Cesme, Turkey, June 17-20 2012, pp. 65-69.

[5] V. Ntranos, M. A. Maddah-Ali, and G. Caire, "Cellular interference alignment," IEEE Trans. on Inf. Theory, vol. 61, no. 3, pp. 1194-1217, March 2015.

[6] A. Sendonaris, E. Erkip, and B. Aazhang, "User cooperation diversity. part i. system description," IEEE Trans. on Communications, vol. 51, no. 11, pp. 1927-1938, Nov 2003.

[7] — , "User cooperation diversity. part ii. implementation aspects and performance analysis," IEEE Trans. on Communications, vol. 51, no. 11, pp. 1939-1948, Nov 2003.

[8] M. Wigger, R. Timo, and S. Shamai (Shitz), "Conferencing in Wyner's asymmetric interference network: Effect of number of rounds," IEEE Trans. on Inf. Theory, vol. 63, Mar. 2017.

[9] F. Willems, "The discrete memoryless multiple access channel with partially cooperating encoders (corresp.)," IEEE Trans. on Inf. Theory, vol. 29 , no. 3, pp. 441-445, May 1983. 